04

\title{
Исследования механизма коммутации короткого вакуумного промежутка с помощью вспомогательного искрового разряда
}

\author{
(ㄷ С.Г. Давыдов, А.Н. Долгов, А.А. Козлов, Р.Х. Якубов \\ Всероссийский научно-исследовательский институт автоматики им. Н.Л. Духова (ВНИИА), \\ 127055 Москва, Россия \\ e-mail: vniia4@vniia.ru
}

Поступило в Редакцию 21 мая 2021 г.

В окончательной редакции 16 июля 2021 г.

Принято к публикации 2 августа 2021 г.

Обнаружена ионизация разреженного газа в коротком промежутке потоком коротковолнового излучения и быстрых электронов из плазмы вспомогательного искрового разряда по поверхности диэлектрика, достаточная для инициирования в промежутке дугового разряда. Плотность ионизирующего потока частиц и излучения определяет время задержки коммутации промежутка по отношению к старту вспомогательного разряда. С увеличением начальной концентрации свободных электронов в основном разрядном промежутке сокращается время перехода к дуговому разряду, обусловленное развитием неустойчивостей.

Ключевые слова: плазма, дуговой разряд, искровой разряд, ионизация, неустойчивость.

DOI: $10.21883 /$ JTF.2021.12.51757.153-21

\section{Введение}

Интерес к искровому разряду по поверхности диэлектрика в вакууме вызван его успешным применением для нужд сильноточной и высоковольтной электроники в приборах коммутации и размыкания цепей [1]. Например, искровой разряд по поверхности диэлектрика используется в качестве инициатора процесса коммутации в вакуумных искровых разрядниках, т.е. для создания проводящей среды в коммутируемом вакуумном промежутке. Обычно полагают, что таковой средой становится непосредственно плазма вспомогательного искрового разряда, формирующаяся из продуктов эрозии материала диэлектрика и электродов.

Цель настоящей работы - выяснить ключевой механизм коммутации короткого вакуумного промежутка, определяющий скорость срабатывания компактного искрового вакуумного разрядника.

\section{1. Эксперименты с макетом коммутатора}

Важный параметр вакуумного искрового разрядника - время задержки между моментом старта вспомогательного искрового разряда-пробоя по поверхности диэлектрика при подаче управляющего импульса напряжения на узел поджига и началом протекания коммутируемого тока через разрядник. Изменение давления в вакуумной камере и расстояния между узлом поджига и основным разрядным промежутком способно привести к существенному изменению времени задержки.

Для проведения экспериментальных исследований был использован открытый макет разрядника, отличающийся тем, что в нем пространственно разделены, но гальванически связаны электродные системы вспомогательного разряда по поверхности диэлектрика (узел поджига) и основного, коммутирующего внешнюю цепь, разряда. Рабочий диапазон давлений остаточного газа составил $10^{-3}-10^{2} \mathrm{~Pa}$. Использованный диапазон расстояний от узла поджига, включающего поджигающий электрод, разделительный диэлектрик и катод № 1, до основного разрядного промежутка, включающего анод и катод № 2, составил 0.25-6.8 cm. Катод № 2 и анод - диски диаметром $5 \mathrm{~mm}$ с отверстием диаметром $3 \mathrm{~mm}$. Протяженность разрядного промежутка между катодом № 2 и анодом равна $1 \mathrm{~mm}$. Толщина диэлектрика $0.1 \mathrm{~mm}$, материал - керамика. Материал электродов нержавеющая сталь (рис. 1).

Для инициирования процесса коммутации на поджигающий электрод подается положительный относительно

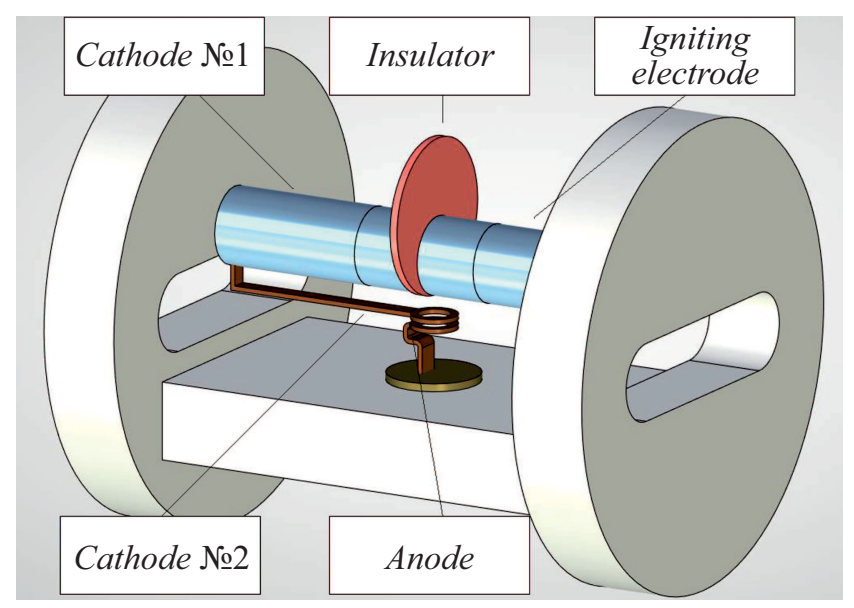

Рис. 1. Исследовательский макет разрядного устройства с двойным катодом. 


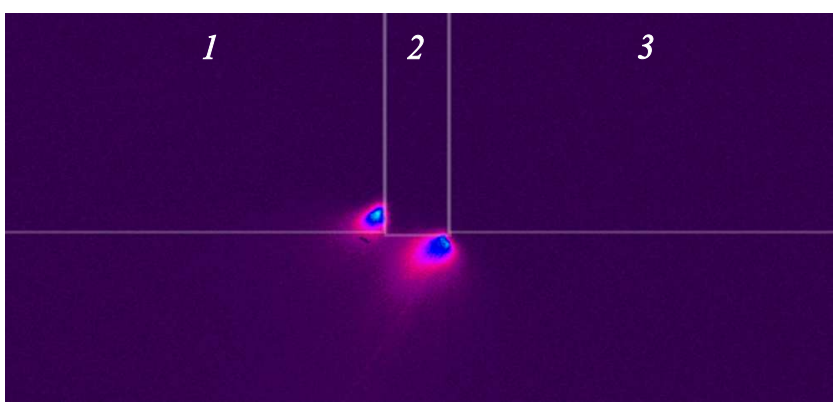

Рис. 2. Изображения искрового разряда в узле поджига макета разрядника, полученные с помощью скоростной фотокамеры в видимом диапазоне спектра: 1 - катод, 2 - диэлектрическя вставка, 3 - поджигающий электрод. Экспозиция $60 \mu \mathrm{s}$. Слева видно яркое катодное пятно и плазму катодного факела, справа - анодное пятно. В промежутке между ними и ниже наблюдается подсвеченная поверхность диэлектрической вставки между поджигающим электродом и катодом № 1.

заземленного катода импульс напряжения амплитудой $3.5 \mathrm{kV}$ и крутизной $(1-2) \cdot 10^{9} \mathrm{~V} / \mathrm{s}$. В результате происходит пробой по цилиндрической поверхности диэлектрика по кратчайшей траектории между прилегающими электродами, и развивается вспомогательный искровой разряд (рис. 2).

Амплитуда силы тока составляет 4-6 А при длительности основной части импульса тока около $20 \mathrm{~ns}$. Плазма, образованная продуктами эрозии, создает проводящую среду в промежутке между катодом № 2 и анодом. Коммутируемый макетом разрядника ток представляет собой однополупериодный синусоидальный импульс тока амплитудой $1.5 \mathrm{kA}$ и продолжительностью $1 \mu \mathrm{s}$. Регистрируемые напряжения в цепи разрядника измерялись с помощью емкостного или резистивного делителя напряжения. Сила регистрируемых токов определялась путем измерения напряжения на низкоиндуктивных сопротивлениях номиналом $0.1-100 \Omega$.

По полученным в экспериментах данным была построена зависимость (рис. 3,a) задержки возникновения дугового разряда, коммутирующего внешнюю цепь, от давления остаточных газов при различных расстояниях от узла поджига до основного разрядного промежутка. Задержка измерялась как временной интервал от момента пробоя по поверхности диэлектрика в узле поджига (момент начала резкого спада напряжения поджига) до момента возникновения коммутируемого тока во внешней цепи.

На графике можно выделить две характерные группы точек: для расстояния $0.25 \mathrm{~cm}$ (задержка $\sim 0.1 \mu \mathrm{s}$ ) и для расстояний $\geq 0.4 \mathrm{~cm}$ (задержка $\sim 1 \mu \mathrm{s}$ ). Наблюдаемые задержки, как нетрудно увидеть, невозможно объяснить только тепловым движением плазмы из узла поджига (тепловая скорость распространения плазмы $\sim 10^{3} \mathrm{~m} / \mathrm{s}$, исходя из ее температуры не более $1.5 \mathrm{eV}$ [2]) или механизмом амбиполярной диффузии плазмы в вакуум (соответствующая скорость $\sim 10^{4} \mathrm{~m} / \mathrm{s}$ [3]). Во-первых, длительность задержки не пропорциональна величине расстояния от узла поджига. Во-вторых, для больших расстояний, по крайней мере, превышающих $3.8 \mathrm{~cm}$, регистрируемое время задержки оказывается слишком мало. Далее, если анализировать ту же зависимость, но уже представленную в полулогарифмическом масштабе (рис. $3, b)$, видно, что зависимость от давления явно выражена для расстояний от $0.4 \mathrm{~cm}$ и более и имеет тенденцию к уменьшению задержки с увеличением давления остаточных газов.

Для больших расстояний замыкание промежутка происходит слишком быстро, чтобы речь шла о замыкании прилетевшей плазмой. Можно предположить, что в начальной стадии процесса коммутации определенную роль играет ионизация остаточного газа коротковолновым излучением и быстрыми электронами (с энергией $\sim 100 \mathrm{eV}[4])$, испущенными из вспомогательного разряда, для чего имеются достаточно веские основания [5-7]. Из-за того что длина свободного пробега как квантов, так и электронов на несколько порядков величины
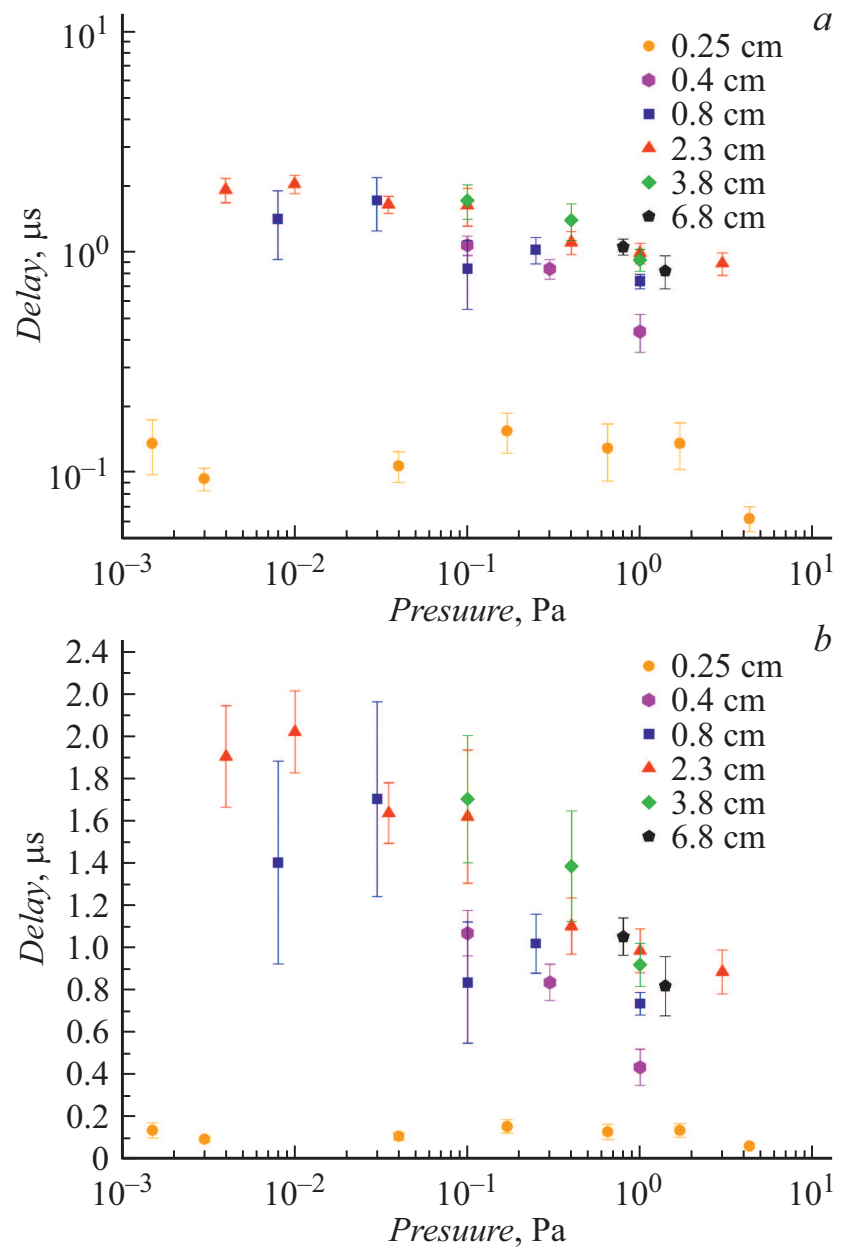

Рис. 3. Зависимость задержки возникновения дугового разряда от давления остаточных газов при различных расстояниях от узла поджига до основного разрядного промежутка: $a-$ в логарифмическом масштабе; $b-$ в полулогарифмическом масштабе. 


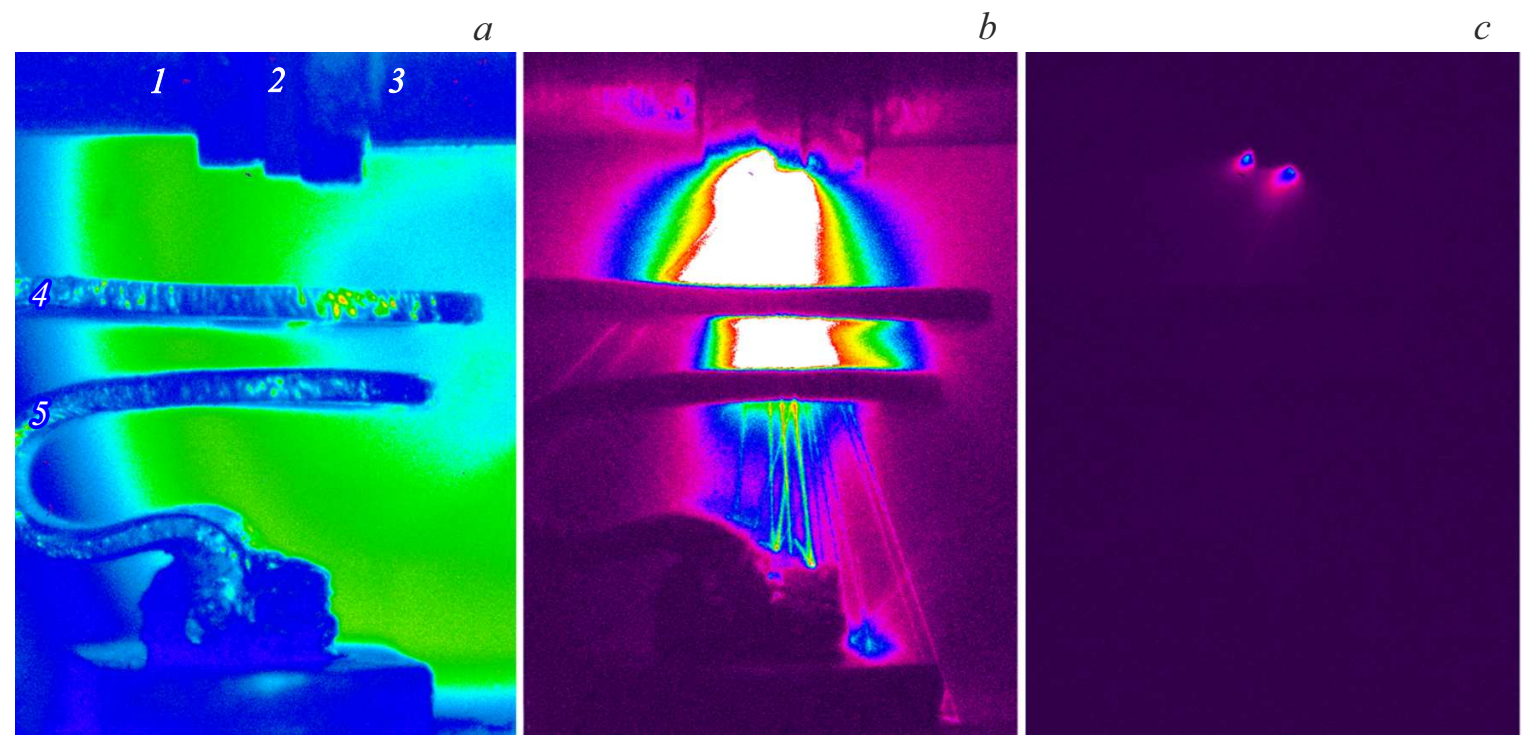

Рис. 4. Фотографии, полученные с помощью скоростной видеокамеры: $a$ - макет разрядника в отсутствие разряда и с подсветкой от внешнего источника: 1 - катод № 1,2 - диэлектрическая вставка, 3 - поджигающий электрод, $4-$ катод № $2,5-$ анод; $b-$ изображение разряда в макете разрядника при положительной полярности потенциала анода $(+100 \mathrm{~V}) ; c-$ изображение разряда в макете разрядника при отрицательной полярности потенциала анода $(-100 \mathrm{~V})$.

превышает размеры разрядного промежутка, в среде слабо ионизованного остаточного газа первоначально возникает слаботочный объемный разряд, например, тлеющий, который претерпевает самопроизвольную контракцию, т.е. сжатие канала тока в результате развития неустойчивости катодного слоя, ионизационноперегревной неустойчивости или неустойчивости иного типа $[8-10]$, и затем переходит в дуговой. Эксперимент показывает, что с увеличением давления остаточных газов происходит уменьшение времени задержки. Это свидетельствует о том, что чем выше начальная концентрация заряженных частиц-носителей тока, обусловленная ионизацией остаточного газа, тем быстрее протекает процесс перехода объемного разряда в дуговой. Причем зависимость носит некоторый пороговый характер: превышение порога начальной концентрации заряженных частиц, связанное с уменьшением расстояния между узлом поджига и основным разрядным промежутком (рис. $3, b)$, приводит к резкому изменению времени задержки и характера зависимости.

Был проведен дополнительный эксперимент, в ходе которого осуществлялась инверсия потенциала анода и производилось скоростное фотографирование разряда в макете разрядника (рис. 4).

Оказалось, что факт зажигания разряда зависит от полярности прикладываемого к электродному промежутку напряжения. При отрицательной полярности анода разряд в основном разрядном промежутке загорался крайне редко. В связи с этим обстоятельством можно сделать вывод о том, что в развитии разряда основную роль играют электроны, эмитируемые узлом поджига. При отрицательном потенциале анода электронам не удается попасть в основной разрядный промежуток и осуществить ионизацию остаточного газа. Следует отметить, что в некоторых случаях разряд все же загорается, по всей видимости, благодаря ионизирующему действию коротковолнового излучения инициирующего разряда.

Сделаем некоторые оценки. При минимальном давлении остаточного газа в наших экспериментах $10^{-3} \mathrm{~Pa}$ концентрация нейтральных частиц в нем составит порядка $n_{0} \approx 10^{17} \mathrm{~m}^{-3}$. Сечение ионизации нейтральных частиц остаточного газа можно принять равным $\sigma_{e} \approx 10^{-20} \mathrm{~m}^{2}$ для ионизации электронным ударом и $\sigma_{p h} \approx 10^{-21} \mathrm{~m}^{2}$ для фотоионизации [4]. Видно, что при прочих равных условиях фотоионизация менее вероятна. Длина свободного пробега для быстрых электронов составит в этом случае $\lambda \approx 1 / n_{0} \sigma_{e} \approx 10^{3} \mathrm{~m}$. При протяженности коммутируемого промежутка $d \approx 10^{-3} \mathrm{~m}$ получим для вероятности ионизирующего столкновения $d / \lambda \approx 10^{-6}$. Количество испускаемых единичным центром взрывной электронной эмиссии и ускоряемых в поле анода электронов оценивается величиной $N \approx 10^{12}$ [3]. Положим, что объем области, в которой в нашем случае происходит эффективная ионизация нейтральных частиц остаточного газа, равен $V \approx d^{3}=10^{-9} \mathrm{~m}^{3}$. Концентрация заряженных частиц, образующихся в остаточном газе под действием быстрых электронов, окажется равной $n \approx(N / V) \cdot(d / \lambda) \approx 10^{15} \mathrm{~m}^{-3}$. Радиус Дебая, если принять $k T \approx 1.5 \mathrm{eV}$, составит $r_{D}=\left(\varepsilon_{0} k T / e^{2} n\right)^{1 / 2} \approx$ $\approx 10^{-4}-10^{-3} \mathrm{~m}<d$, где $\varepsilon_{0}$ - диэлектрическая постоянная, $k$ - постоянная Больцмана, $T-$ температура хаотического движения свободных электронов, $e-$ заряд электрона. Таким образом, при давлениях остаточного 
газа $\geq 10^{-3}$ Ра вполне возможно образование описанным выше путем проводящей среды - плазмы. Однако если посчитать скорость токового дрейфа электронов, исходя из необходимой для возникновения единичного центра взрывной эмиссии электронов силы тока $\sim 10 \mathrm{~A}[3]$, то она окажется слишком велика, т.е. много больше скорости ионного звука. Следовательно, в плазме будут возбуждаться ленгмюровские колебания, на которых будут рассеиваться проводящие ток электроны, что обеспечит рост тепловой мощности, выделяющейся в канале тока, рост тока термоэмиссии, потоки плазмы с катода и анода. Возникнут условия для размножения центров взрывной электронной эмиссии. Захват в плазму токового канала нескольких электронов, испускаемых этими центрами, уже способен создать условия для образования катодного пятна и развития искрового разряда. Таким образом, увеличение мощности, вкладываемой во вспомогательный разряд, способно ускорить переход к самостоятельному сильноточному разряду в коммутируемом промежутке за счет повышения начальной концентрации свободных электронов в результате ионизации остаточного газа. А вот увеличения концентрации свободных электронов за счет разлета плазмы из области поверхностного разряда можно ожидать только при расстояниях от узла поджига до основного разрядного промежутка не более $0.25 \mathrm{~cm}$.

\section{2. Эксперименты с компактным вакуумным искровым разрядником}

Плотность потока ионизирующего излучения и быстрых электронов из плазмы вспомогательного разряда можно попытаться увеличить путем увеличения энергии, вкладываемой во вспомогательный разряд. Таким образом можно повлиять на задержку срабатывания разрядника в направлении ее уменьшения и компенсировать ее зависимость от давления остаточного газа.

Соответствующие исследования были проведены с использованием компактных управляемых вакуумных разрядников, которые представляют собой трехэлектродную коаксиальную систему, основными элементами которой являются анод, катод, поджигающий электрод и диэлектрическая шайба, разделяющая катод и поджигающий электрод (рис.5). Разрядное устройство размещается в отпаянном герметичном диэлектрическом корпусе, откачиваемом до давления не хуже $10^{-1} \mathrm{~Pa}$.

Электроды были выполнены из алюминиевого сплава, диэлектрическая шайба выполнялась из слюды толщиной $0.1 \mathrm{~mm}$. Диаметр внутреннего цилиндрического электрода (катод) составляет $5 \mathrm{~mm}$. Внешний электрод (анод) выполнен в виде полого цилиндра и имеет внутренний диаметр $7 \mathrm{~mm}$. Между диэлектрической шайбой и прилегающими электродами соосно с ними размещаются дополнительные металлические втулки, каждая толщиной $0.1 \mathrm{~mm}$ и соответствующего диаметра, между диэлектрической шайбой и поджигающим электродом -



Рис. 5. Принципиальная конструкция компактного вакуумного искрового разрядника: 1 - диэлектрическая герметичная оболочка разрядника, 2 - поджигающий электрод, 3 - анод, 4 - катод, 5 - вспомогательные металлические втулки, 6 - диэлектрическая шайба.

коваровая, между диэлектрической шайбой и катодом коваровая и титановая. Поджигающий электрод, ближайшая к нему кромка катода, дополнительные металлические прокладки и диэлектрическая шайба вместе составляют узел поджига.

В качестве испытуемого образца был выбран разрядник, функционирующий в режиме с напряжением $1.5 \mathrm{kV}$ на промежутке катод-анод. При использовании ранее описанной схемы поджига задержка тока, коммутируемого разрядником, хаотично изменялась в интервале от 200 до 1500 ns. Изменяя, в первую очередь, номинал емкостного накопителя энергии удалось увеличить достигаемую в узле поджига силу тока до $110 \mathrm{~A}$. Энергия в емкостном накопителе узла поджига при этом варьировалась от $5 \cdot 10^{-5}$ до $6 \cdot 10^{-2} \mathrm{~J}$. Энергия, запасаемая во внешней коммутируемой цепи, составляла неизменно $0.6 \mathrm{~J}$. С ростом энергии в схеме поджига происходило затягивание вспомогательного разряда. Результаты исследований свидетельствуют о том, что увеличение энергии, вкладываемой во вспомогательный разряд на стадии искры, дает наиболее ощутимое уменьшение времени задержки. Энергия, вложенная во вспомогательный разряд, определялась путем расчета на основе измерений динамики тока и напряжения в разряде.

Для анализа фиксируемых изменений задержки были построены диаграммы распределения задержки при разных уровнях энергии схемы поджига. Диаграммы представляют собой функции распределения задержек, нормированные на полное число коммутаций (рис. 6). Они позволяют визуально оценить величину задержки и ее стабильность, а также наглядно увидеть измене- 
ние функции распределения задержек в зависимости от уровня энергии схемы поджига.

Проделанные измерения показали, что удается путем повышения используемой энергии поджига не только уменьшать время задержки срабатывания разрядника (рис. 7), но и сделать процесс функционирования разрядника более стабильным в результате уменьшения разброса регистрируемых значений указанной величины.

В то же время результаты проведенных исследований показали, что степень воздействия на время задержки падает с увеличением продолжительности импульса протекающего по поверхности диэлектрика тока (рис. 8). Последнее обстоятельство, по-видимому, связано с тем, что с увеличением продолжительности вспомогательного разряда увеличивается относительная продолжительность его дуговой стадии, переход к которой сопровождается быстрым падением мощности, выделяющейся в канале разряда.

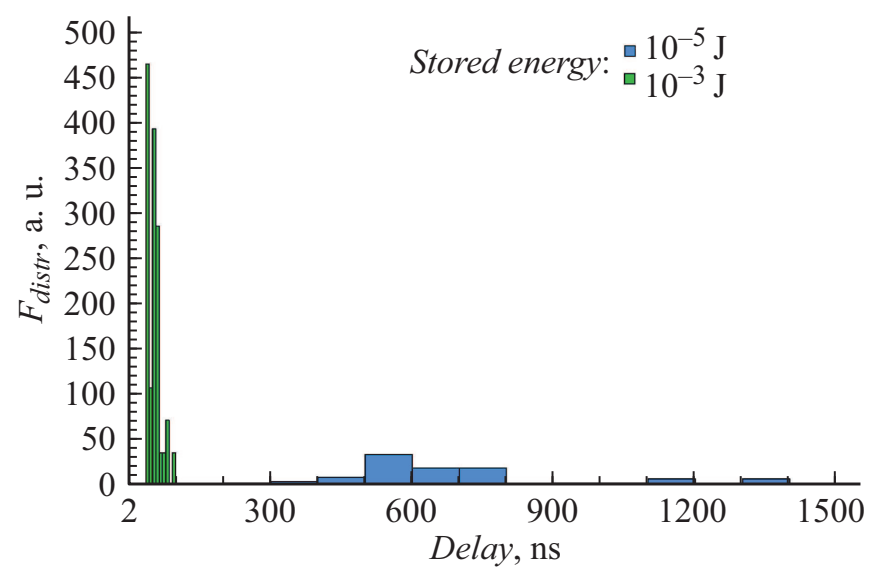

Рис. 6. Сравнение диаграмм распределения задержек для схемы поджига с энергией в накопителе $E=1 \cdot 10^{-5} \mathrm{~J}$ и схемы c $E=1 \cdot 10^{-3} \mathrm{~J}$.

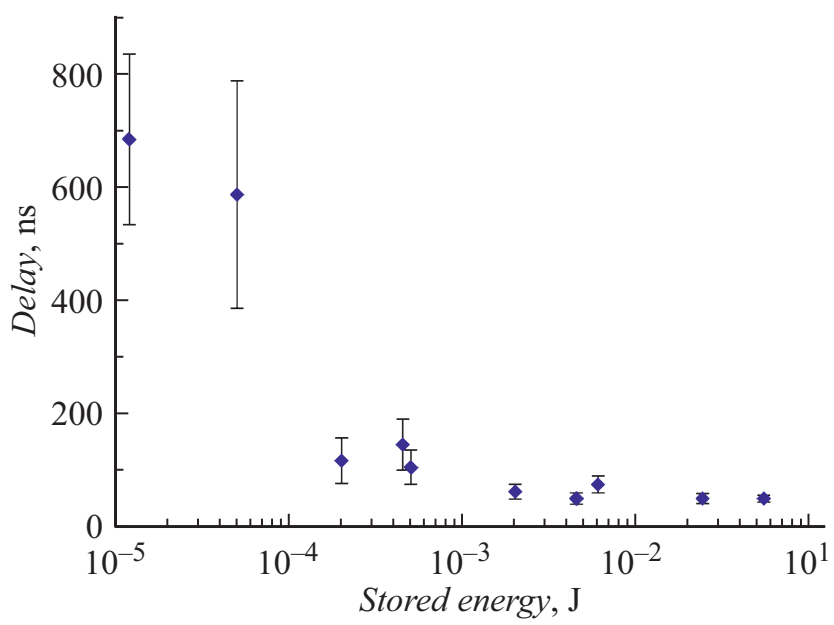

Рис. 7. Зависимость задержки возникновения дугового разряда в промежутке катод-анод от энергии, запасенной в схеме поджига.

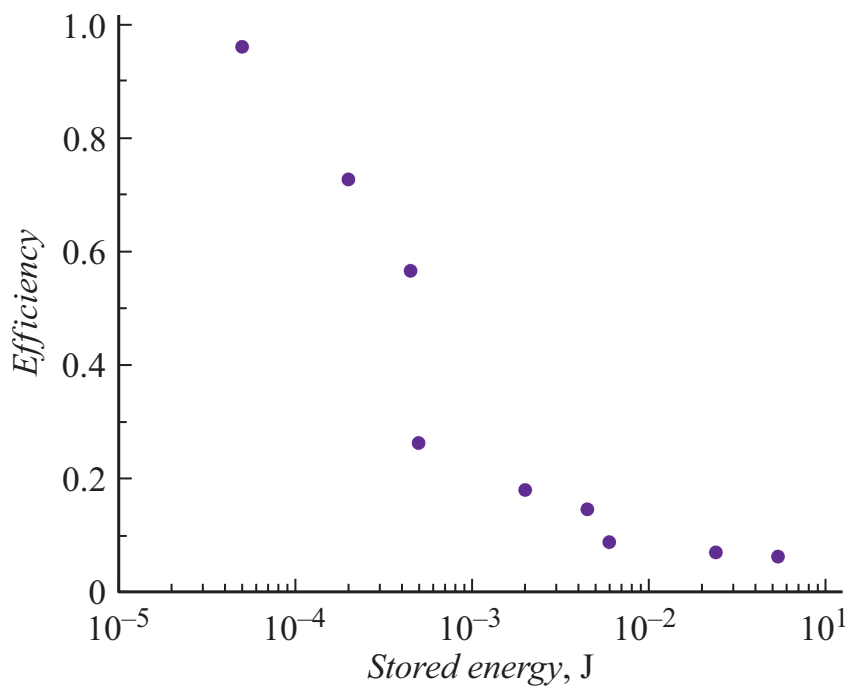

Рис. 8. Диаграмма зависимости доли энергии, вложенной в инициирующий процесс коммутации разряда, от энергии, запасенной в схеме поджига.

\section{3. Заключение}

На основе анализа полученных экспериментальных данных процесс коммутации короткого вакуумного промежутка с помощью вспомогательного искрового разряда может быть представлен следующим образом. На начальной стадии происходит ионизация остаточного газа в коммутируемом промежутке коротковолновым излучением и быстрыми электронами из плазмы вспомогательного разряда с преобладанием ионизации электронным ударом. При начальной концентрации свободных носителей заряда ниже некоторой пороговой, первоначально, вероятно, возникает слаботочный объемный разряд, который затем испытывает контракцию и превращается в искровой, переходящий в дугу. При начальной концентрации свободных электронов выше пороговой становится возможным возникновение искрового разряда без промежуточной стадии.

С увеличением энергии, подводимой к схеме поджига малогабаритного вакуумного искрового разрядника, наблюдается устойчивое снижение времени задержки срабатывания разрядника и повышение уровня стабильности задержки. Данное обстоятельство можно связать с увеличением начальной концентрации свободных электронов в основном разрядном промежутке и соответствующим сокращением времени перехода к самостоятельному сильноточному разряду.

Наиболее эффективное с точки зрения минимизации и стабильности времени задержки срабатывания разрядника вложение энергии в формирование инициирующей плазмы происходит на искровой стадии вспомогательного разряда по поверхности диэлектрика в узле поджига. 


\section{Конфликт интересов}

Авторы заявляют, что у них нет конфликта интересов.

\section{Список литературы}

[1] A. Anders. Cathodic Arcs (Springer Series on Atomic, Optical, and Plasma Physics, NY., 2008), DOI: $10.1007 / 978-0-387-79108-1$

[2] A. Batrakov, S. Popov, N. Vogel, B. Juttner. IEEE Transactions on Plasma Science, 31 (5), 817 (2003).

DOI: $10.1109 /$ TPS.2003.818427

[3] Г.А. Месяц. Взрывная электронная эмиссия (Физматлит, M., 2011)

[4] А.П. Бабичев, Н.А. Бабушкина, А.М. Братковский, М.Е. Бродов, М.В. Быстров, Б.В. Виноградов, Л.И. Винокурова, Э.П. Гельман, А.П. Геппе, И.С. Григорьев, К.Г. Гуртовой, В.С. Егоров, А.В. Елецкий, Л.К. Зарембо, В.Ю. Иванов, В.Л. Ивашинцева, В.В. Игнатьев, Р.М. Имамов, А.В. Инюшкин, Н.В. Кадобнова, И.И. Карасик, К.А. Кикоин, В.А. Криворучко, В.М. Кулаков, С.Д. Лазарев, Т.М. Лифшиц, Ю.Э. Любарский, С.В. Марин, И.А Маслов, Е.3. Мейлихов, А.И. Мигачев, С.А. Миронов, А.Л. Мусатов, Ю.П. Никитин, Л.А. Новицкий, А.И. Обухов, В.И. Ожогин, Р.В. Писарев, Ю.В. Писаревский, В.С. Птускин, А.А. Радциг, В.П. Рудаков, Б.Д. Сумм, Р.А. Сюняев, М.Н. Хлопкин, И.Н. Хлюстиков, В.М. Черепанов, А.Г. Чертов, В.Г. Шапиро, В.М. Шустряков, С.С. Якимов, В.П. Яновский. Физчческие величины: Справочник, под ред. И.С. Григорьева, Е.З. Мейлихова. (Энергоиздат, М., 1991)

[5] С.Г. Давыдов, А.Н. Долгов, А.В. Корнеев, Р.Х. Якубов. Письма в ЖТФ, 45 (12), 33 (2019). DOI: 10.21883/JTF.2021.12.51757.153-21

[6] С.Г. Давыдов, А.Н. Долгов, А.С. Каторов, В.О. Ревазов, P.Х. Якубов. Прикладная физика, 1, 39 (2021). DOI: 10.51368/1996-0948-2021-1-39-43

[7] H.C. Miller. IEEE Transactions on Dielectrics and Electrical Insulation, 22 (6), 3641 (2015). DOI: 10.1109/14.231534

[8] Райзер Ю.П. Физика газового разряда (Наука, М., 1992).

[9] S.A. Barentgolts, D.L. Shmelev, I.V. Uimanov. IEEE Transactions on Plasma Science, 43 (8), 2236 (215). DOI: 10.1109/TPS.2015.2431321

[10] Е.В. Паркевич, М.А. Медведев, А.И. Хирьянова, Г.В. Иваненков, А.В. Агафонов. В сб.: Электрофизические и оптические процессы в плазменных и твердотельных средах и наноструктурах, под ред. Г.А. Месяца. (РУСАЙНС, М., 2019), с. 217. 